\title{
Epidemiology and clinical management of XDR-TB: a systematic review by TBNET
}

\author{
G. Sotgiu, G. Ferrara, A. Matteelli, M.D. Richardson, R. Centis, S. Ruesch-Gerdes, \\ O. Toungoussova, J-P. Zellweger, A. Spanevello, D. Cirillo, C. Lange and G.B. Migliori
}

ABSTRACT: Extensively drug-resistant tuberculosis (XDR-TB) is present in all regions and poses serious challenges for public health and clinical management. Laboratory diagnosis is difficult and little evidence exists to guide clinicians in treating people with XDR-TB effectively. To summarise the available data on diagnosis and treatment, the current authors performed a systematic review on 13 recent studies of the epidemiology and clinical management of XDR-TB.

Studies that met inclusion criteria were reviewed, in order to assess methodology, treatment regimens and treatment outcomes.

Meta-analysis of currently available data is not possible because of inconsistent definitions and methodologies. Data show that XDR-TB can be successfully treated in up to $65 \%$ of patients, particularly those who are not co-infected with HIV. However, treatment duration is longer and outcomes are in general poorer than for non-XDR TB patients.

To strengthen the evidence for extensively drug-resistant tuberculosis diagnosis, treatment and prevention, future studies should: 1) be prospective in design; 2) adopt standardised, internationally accepted definitions; 3) use quality-assured laboratory testing for all first- and second-line drugs; and 4) collect data on an agreed-upon set of standard variables, allowing for comparisons across studies. Early diagnosis and aggressive management of extensively drugresistant tuberculosis provide the best chance of positive outcome, but prevention is still paramount.

KEYWORDS: Extensively drug-resistant tuberculosis, microbiological diagnosis, outcomes, systematic review, treatment efficacy

\begin{abstract}
A ccording to the latest World Health Organization (WHO) estimates, approximately 9.2 million new cases of tuberculosis (TB) and 1.5 million TB-related deaths occurred in 2006. TB remains a global emergency [1]. TB treatment requires multiple antibiotics over 6 months or more to achieve cure but, for decades, no new and better drugs have been developed and licensed. In addition, drug-resistant strains of Mycobacterium tuberculosis have emerged as a serious problem. The prevalence of multidrugresistant (MDR) M. tuberculosis (defined as in vitro resistance to isoniazid and rifampicin, the two most potent first-line drugs for TB treatment) is now widely reported $[2,3]$. MDR strains are currently found in more than $15 \%$ of all new cases
\end{abstract}

of TB in some areas of the former Soviet Union (Azerbaijan, Moldova, Ukraine and Tomsk Oblast (Russian Federation)), and in more than $10 \%$ in parts of China and other areas of the former Soviet Union (Latvia, Estonia, Kazakhstan, Uzbekistan, and Ivanovo and Mari El (both Russian Federation)) [4-6].

Strains of $M$. tuberculosis resistant to second-line drugs are also emerging. Cases of extensively drug-resistant (XDR)-TB (defined as in vitro drug resistance to isoniazid and rifampicin plus any fluoroquinolone and at least one of the injectable drugs (capreomycin, kanamycin or amikacin)) have been described around the globe $[7,8]$. Adverse treatment outcomes for complicated

Earn CME accreditation by answering questions about this article. You will find these at the back of the printed copy of this issue or online at www.erj.ersjournals.com/current.dtl

AFFILIATIONS

For affiliations please see the Acknowledgements section.

CORRESPONDENCE

G.B. Migliori

WHO Collaborating Centre for TB and Lung Diseases, Fondazione S Maugeri, Care and Research Institute TBNET Secretariat (Tuberculosis Network European Trials Group) Via Roncaccio 16 21049

Tradate Italy

Fax: 390331829402

E-mail: giovannibattista.migliori@ fsm.it

Received:

November 062008 Accepted after revision: January 122009

STATEMENT OF INTEREST None declared. 
MDR-TB cases (those with additional resistance beyond isoniazid and rifampicin) and cases with XDR-TB occur more frequently than for other cases of TB with lower levels of drug resistance [9-25].

Despite the growing amount of public awareness about TB drug resistance, the essential variables necessary to fully understand XDR-TB are not systematically collected, analysed and reported in published studies. These essential variables include clinicalepidemiological and demographic features of cases enrolled, details of how drug-susceptibility testing (DST) was performed, treatment regimens and treatment outcomes [25].

To the current authors' knowledge, despite its clinical and public health interest, no review article on this topic had been published at the time of writing (December 15, 2008). The aim of the present systematic review of available literature on XDRTB is to assess methodological challenges in studying XDR-TB, summarise current knowledge, and suggest ways to strengthen the global body of knowledge on the clinical epidemiology of XDR-TB, thus enhancing our ability to control and prevent this global threat to public health.

\section{METHODS}

\section{Study design}

The current study is a systematic review of published Englishlanguage literature on the clinical epidemiology, diagnosis, treatment approaches and outcomes of XDR-TB, according to the Cochrane guidelines [26].

\section{Selection criteria}

Two independent researchers used the same keywords and methodology to generate a list of original research articles published in peer-reviewed journals in clinical epidemiology and clinical management of XDR-TB. Using Medline and Embase databases, they searched for terms describing $M$. tuberculosis drug resistance (keywords: "tuberculosis", "extensively drug-resistant tuberculosis", "XDR-TB", "multidrugresistant tuberculosis" and "MDR-TB"). Potentially relevant articles were retrieved and the reference lists were reviewed, to identify studies that the search strategy may have missed.

\section{Final inclusion criteria}

Abstracts of journal articles were reviewed to retrieve only those containing: 1) a definition of XDR-TB consistent with the revised, current definition of XDR-TB (as stated in the document published by the Centers for Disease Control and Prevention in 2006 [8] and in other relevant articles [7, 27]); 2) a cohort of $>100$ MDR-TB cases from which data were gathered on XDR-TB cases; 3) evaluation of treatment regimens or of treatment outcomes (as proposed by LASERSON et al. [28] or, alternatively, by the WHO [1]); and 4) a publication date between March 1, 2006 and December 15, 2008. Each study included was systematically reviewed in terms of methodology, assumptions, results and conclusions, setting the current review apart from other recent overviews of XDR-TB.

Formal meta-analytic techniques could not be applied for a comparative analysis because the studies identified used different measures (i.e. mean or median, standard deviation or interquartile range) and some did not report parameter estimates.

\section{Evaluation criteria}

The following topics were reviewed and discussed by at least three independent researchers, to be then approved by the whole research panel.

\section{Clinical and epidemiological features of cases enrolled}

All selected studies were reviewed to extract the following data regarding the clinical and epidemiological features of cases enrolled: 1) the drugs tested to define XDR-TB cases; 2) the features of the cohort, including the number of MDR and XDR cases; 3 ) the proportion of cases previously treated $>1$ month; 4) the number of previous treatment regimens longer than 1 month; 5) the number of drugs to which the strain was resistant; 6) the prevalence of HIV-positive cases in the studied cohort; and 7) the representativeness of the sample based on enrolment criteria and national coverage. The sample was considered representative for the purpose of discussing clinical features when the majority (defined as $>65 \%$ ) of MDR-TB cases diagnosed at national level were included in the study.

\section{Laboratory diagnosis}

All selected studies were reviewed to extract the following data regarding the microbiological diagnosis of MDR- and XDR-TB: 1) quality assurance for DST, defined as laboratories undergoing proficiency testing from supranational reference laboratories (SRLs) or SRLs themselves [29]; and 2) the mean number of second-line drugs tested, with particular attention to the drugs needed to define a strain as MDR or XDR.

\section{Characteristics of treatment}

All selected studies were reviewed to extract the following data regarding treatment: 1) drugs used (individualised or standardised treatment), 2) availability of first-, second- and third-line drugs, particularly availability of linezolid; 3) use of surgery; and 4) reporting of adverse events of drug treatment.

\section{Treatment efficacy end-points}

All selected studies were reviewed to extract the following data regarding the clinical effectiveness end-points of the evaluated cases associated with treatment regimens: 1 ) time to sputum smear and culture conversion; 2) proportion of treatment success (individuals cured and those who completed treatment), failure and death, using the criteria of LASERSON et al. [28]. Time of follow-up was evaluated in the articles reviewed. Only the end-points related to MDR/XDR-TB cases were considered in the review.

\section{References}

The references included in each study reviewed were checked to see if all the relevant studies (e.g. those selected by the current review) were quoted.

\section{RESULTS}

The Medline search generated 228 articles and Embase generated 236 articles related to XDR-TB. Of these, 13 met the inclusion criteria and are presented in the current review.

Clinical and epidemiological features of cases enrolled The clinical and epidemiological features of cases enrolled are shown in tables 1 and 2. All 13 studies included in the review 
TABLE 1 Clinical and epidemiological features of cases enrolled in the studies reviewed

\begin{tabular}{|c|c|c|c|c|c|c|}
\hline \multirow[t]{2}{*}{ First author [Ref.] } & \multicolumn{3}{|c|}{ Total MDR cohort } & \multirow[t]{2}{*}{ MDR-TB n } & \multirow[t]{2}{*}{ XDR-TB n } & \multirow[t]{2}{*}{ Representative sample ${ }^{\#}$} \\
\hline & Subjects $\mathrm{n}$ & Location & Year & & & \\
\hline GANDHI [9] & 221 & $\begin{array}{c}\text { Msinga sub-district of } \\
\text { KwaZulu Natal, South } \\
\text { Africa }\end{array}$ & 2005-2006 & 168 & 53 & No \\
\hline Kıм [12] & 211 & $\begin{array}{c}\text { Seoul University Hospital, } \\
\text { Korea }\end{array}$ & 1996-2005 & 168 & 43 & No \\
\hline MITNICK [16] & 651 & Lima, Peru & 1999-2002 & 603 & 48 & Yes \\
\hline Kwon [20] & 155 & Seoul, Korea & 1995-2004 & 128 & 27 & No \\
\hline LAI [21] & 160 & Taiwan & $2000-2006$ & 150 & 10 & No \\
\hline BANERJEE [22] & 424 & California, USA & 1993-2006 & 406 & 18 & Yes: $100 \%$ \\
\hline BONILLA [23] & 1989 & Peru & 1997-2007 & 1870 & 119 & Yes" \\
\hline Kıм [24] & 1407 & South Korea & 2000-2002 & 1332 & 75 & Yes" \\
\hline Median & 221 & & & 177 & 29 & \\
\hline
\end{tabular}

MDR: multidrug-resistant; TB: tuberculosis; XDR: extensively drug-resistant. ${ }^{\#}$ : MDR coverage $>65 \%$; ${ }^{\text {: }}$ the sample was considered representative according to the information available in the article.

used a definition of XDR-TB consistent with the current WHO definition (in vitro drug resistance to isoniazid and rifampicin plus to any fluoroquinolone and at least one of the injectable drugs (capreomycin, kanamycin or amikacin)) [7, 8]. Out of the 13 studies, eight $(62 \%)$ performed DST on all three injectable drugs included in the XDR-TB definition $[7,8]$ and five on only one of the three injectables. In those five studies, therefore, additional cases of XDR-TB may not have been identified because of the lack of comprehensive DST.

All 13 studies reported the number of MDR- and XDR-TB cases. Overall cohort size ranged from 1,989 MDR-TB cases (including 119 XDR-TB cases) in Peru [23] to 137 MDR-TB cases (including 11 XDR-TB cases) in several European countries [10]. The proportion of XDR-TB cases among MDRTB cohorts ranged from $24 \%$ in South Africa [9] to $4 \%$ in Germany [18], but a number of them were not representative of the overall MDR-TB population in the country.

The proportion of retreatment cases was reported in $10(77 \%)$ out of 13 studies. The proportion of retreatment cases among MDR-TB cases ranged 49-98\% (being higher in Tomsk (Russia), Peru and Korea [16, 19, 20, 23, 24] than in Germany, Italy, Estonia and Russia [10, 11]). The proportion of retreatment cases among XDR-TB cases ranged $45-100 \%$, being consistently higher in XDR- than in MDR-TB cases.

Only six (46\%) studies reported the mean or median number of previous treatment regimens longer than 1 month. It was consistently higher among XDR-TB cases (range 2-4.2) than among MDR-TB cases (1.6-3.2).

Only five (39\%) out of 13 studies reported the number of drugs to which strains of $M$. tuberculosis were resistant. Three studies reported the median number and two the mean number of drugs to which strains demonstrated in vitro resistance. In all of these studies, the number of drugs to which a strain of $M$. tuberculosis was resistant was higher in XDR-TB cases (range 79) than in MDR-TB cases (4-5.3). The sample was considered representative in eight $(62 \%)$ studies, with a population coverage ranging $65-100 \%$.

The report detailing the Tugela Ferry outbreak of XDR-TB in South Africa was the only study concerning HIV-infected patients [9], while the remaining reported HIV-uninfected subjects almost exclusively. HIV testing practices were not described in the majority of studies. The prevalence of HIV in the studied cohorts was $<5 \%$ in the all studies except the one from South Africa (in which it was 100\%) [9].

\section{Laboratory diagnosis}

Microbiological features of the studies reviewed are shown in table 3. Out of the 13 studies included in the review, eight $(62 \%)$ reported that DST was performed for all second-line drugs necessary to diagnose XDR-TB. In six $(46 \%)$ of the studies, the diagnosis of MDR/XDR-TB was based on DST performed under SRL quality control or in SRLs themselves. No data were available on the methods used for cultures (solid versus liquid media) or on DST to assess the resistance of the 
TABLE 2 Clinical and epidemiological features of cases enrolled in the studies reviewed

\begin{tabular}{|c|c|c|c|c|c|c|c|c|c|c|}
\hline \multirow[t]{2}{*}{ First author [Ref.] } & \multicolumn{2}{|c|}{ Previously treated } & \multicolumn{2}{|c|}{$\begin{array}{l}\text { Previous } \\
\text { treatment } \\
>1 \text { month }\end{array}$} & \multicolumn{2}{|c|}{ Drugs used } & \multicolumn{2}{|c|}{$\begin{array}{c}\text { Cohort prevalence of } \\
\text { HIV }\end{array}$} & \multirow[t]{2}{*}{ Linezolid } & \multirow[t]{2}{*}{ Definitions $^{\#}$} \\
\hline & MDR & XDR & MDR & XDR & MDR & XDR & MDR & XDR & & \\
\hline GANDHI [9] & & $21(45)$ & & & & & & 44 (100) & & WHO \\
\hline Kıм [12] & $103(61.3)$ & $24(55.8)$ & & & $6^{*}$ & $7^{\circ}$ & $0(0)$ & $0(0)$ & Yes & LASERSON \\
\hline MITNICK [16] & $47(97.9)$ & $602(99.8)$ & $3.2^{+}$ & $4.2^{+}$ & & 5.3 & $9(1.5)$ & $0(0)$ & & LASERSON \\
\hline \multicolumn{11}{|l|}{ СHAN [17] } \\
\hline KeShaVJee [19] & $574(95.5)$ & $29(100)$ & $2^{*}$ & $3^{\circ}$ & & & $5(0.9)$ & $0(0)$ & & LASERSON \\
\hline EKER [18] & $94(53)$ & $6(86)$ & $2^{\circ}$ & $2.4^{\circ}$ & $4^{+}$ & $5^{+}$ & $7(4.9)$ & $0(0)$ & Yes & LASERSON \\
\hline KwON [20] & $113(88)$ & $24(89)$ & & & $6^{\circ}$ & $6^{\prime}$ & $0(0)$ & $0(0)$ & & LASERSON \\
\hline BONILLA [23] & $88.9^{5}$ & $88.5^{\S}$ & 1.6 & 2 & & & & & & LASERSON \\
\hline Кıм [24] & 1286 (96) & $73(97)$ & & & $5^{\circ}$ & $5^{\circ}$ & $0(0)$ & $1(1.7)$ & & WHO \\
\hline
\end{tabular}

Data are presented as $\mathrm{n}$ or $\mathrm{n}(\%)$, unless otherwise stated. MDR: multidrug-resistant; XDR: extensively drug-resistant; WHO: World Health Organization. ${ }^{\#}$ : according to LASERSON et al. [28] and/or the WHO; ${ }^{\bullet}$ : median; ${ }^{+}$: mean; ${ }^{\varsigma}$ : data presented as \%.

strains reported in the studies. Altogether, 148 (35\%) out of 428 XDR-TB patients and 1,720 (36\%) out of 4,774 MDR-TB patients received a diagnosis based on quality-controlled DST.

Five $(38 \%)$ studies reported the number of first- and the second-line drugs tested and their results: strains were resistant to a mean or median of five and eight drugs in patients diagnosed as MDR- and XDR-TB, respectively.

\section{Characteristics of treatment}

The characteristics of treatment are shown in table 4. Although the majority of the studies reported the use of individualised treatment regimens [10-12, 16, 18-20, 22-24], two cohorts were prescribed standardised treatment regimens [9, 23]. First-line drugs were reported to be available in all studies, and the vast majority of cohorts had access to all second-line drugs [9-12, 16-24]. Linezolid was reported to be available in only three

TABLE 3 Microbiological features in the studies reviewed

First author [Ref.]

Drugs to which strains were resistant $\mathbf{n}$

XDR

GANDHI [9]

MIGLIORI [10]

MigLIORI [11]

КІм [12]

MitNICK [16]

Chan [17]

Keshavjee [19]

EKER [18]

KWON [20]

LAI [21]

BANERJee [22]

BONILLA [23]

КıM [24]
$100 \%$ SRL QA DST

All drugs tested

MDR: multidrug-resistant; XDR: extensively drug-resistant; SRL: supranational reference laboratory; QA: quality assurance; DST: drug susceptibility testing. ${ }^{*}$ : median; mean. 
TABLE 4 Characteristics of treatment in the studies reviewed

First author [Ref.]

Standard or individualised treatment

Surgery reported Adverse events reported

\begin{tabular}{|c|}
\hline GANDHI [9] \\
\hline MIGLIORI [10] \\
\hline MIGLIORI [11] \\
\hline Kıм [12] \\
\hline MITNICK [16] \\
\hline Chan [17] \\
\hline KeShavJeE [19] \\
\hline EKER [18] \\
\hline KwoN [20] \\
\hline LAI [21] \\
\hline BANERJEE [22] \\
\hline BONILLA [23] \\
\hline Кıм [24] \\
\hline
\end{tabular}

Standard
Individualised
Individualised
Individualised
Individualised
Individualised
Individualised
Individualised
Individualised
Standard or individualised or empirical
Individualised

$\begin{array}{ll}\text { No } & \text { No } \\ \text { No } & \text { No } \\ \text { No } & \text { No } \\ \text { Yes } & \text { Yes } \\ \text { Yes } & \text { No } \\ \text { Yes } & \text { No } \\ \text { Yes } & \text { Yes } \\ \text { Yes } & \text { Yes } \\ \text { Yes } & \text { No } \\ \text { No } & \text { No } \\ \text { No } & \text { No } \\ \text { No } & \text { No } \\ \text { No } & \text { No }\end{array}$

studies $[12,18,21]$; therefore, data were insufficient to comment further on its efficacy and safety.

The rate and nature of adverse events during XDR-TB treatment were mentioned in some studies, none of them suggesting that adverse events are an important limiting factor for the treatment of XDR-TB compared with MDR-TB cases $[12,18,19]$.

\section{Treatment efficacy end-points}

The treatment efficacy end-points are shown in table 5. Out of the 13 studies included in the review, only three $(23 \%)$ evaluated both median time to sputum smear and culture conversion, while four measured only the median time to culture conversion.

Longer treatment duration and delayed sputum smear conversion were reported in most studies among XDR-TB patients. Median time to sputum smear conversion ranged 4156 days in MDR-TB patients, while it ranged $88-110$ days in XDR-TB cases. The median time to culture conversion ranged 58-99 days in MDR-TB cases; it was substantially different in XDR-TB patients, ranging 60-195 days.

There were 11 studies reporting on treatment outcome in a series of XDR-TB cases [9-12, 16, 18-20, 22-24]. Patients were followed up for a minimum of 18 months in the studies in which it was reported. Out of the 13 studies, four (31\%) used the WHO definition of cure $[9,10,22,24]$, six $(46 \%)$ used the LASERSON et al. [28] definition of cure [12, 16, 18-20,23] and two did not specify the definition used $[17,21]$. Although one study used the WHO definition [11], its cohort data were reevaluated using the LASERSON et al. [28] definition in order to improve comparability [13].

Analysis of treatment outcomes among HIV-infected individuals in the South Africa report revealed that $98 \%$ (52 out of 53) died with a median survival time of only 16 days [9]. However, it is doubtful whether this finding can be generalised to all HIV-seropositive individuals with XDR-TB, and the outcome was certainly better in studies of predominantly HIVseronegative individuals, where the proportion of treatment success exceeded $48 \%$ in all cohorts. Seven studies reported on the use of surgery, which varied from 4 to $56 \%$ of XDR-TB patients $[12,16-20,24]$. In two studies, higher success rates were associated with the use of surgery among XDR-TB patients $[16,20]$.

The studies reviewed provide evidence against the statement that XDR-TB cases are virtually untreatable. In nine studies that provided figures on treatment success, the overall success rate (cured and completed) ranged from $34 \%$ [11] to $67 \%$ [20]. The success rate of XDR-TB cases was compared with that of MDR-TB cases in 10 studies: no difference was found in five $[11,12,16,18,20]$, while in the remaining five the success rate was significantly reduced among XDR-TB cases, by $\sim 1.5$ times [23] up to 20 times [17].

The proportion of cases failing their treatment was only assessed in seven $(54 \%)$ studies; it ranged $10.4-31 \%$ in the XDR-TB cases and $0.6-21 \%$ in MDR-TB patients. The majority of the nine studies reporting on death also demonstrated a high mortality rate among XDR-TB cases, ranging from 7\% [19] to $36 \%$ [10], with the rate being $>20 \%$ in five studies [10, 16 , 22-24]. Mortality was compared with that of MDR-TB cases in nine studies: three found similar rates $[16,19,23]$ and the remaining six found increased mortality among XDR-TB cases: from a two-fold $[12,18,22]$ up to a five-fold increase [10]. The relative risk for death due to XDR-TB (compared with MDRTB) was found in the study by MigLiori et al. [11] to be 5.5, and was computed based on the data of KIM et al. [12], who found this relative risk to be 1.8 .

One study reported that XDR-TB cases that were initially treated on the basis of the DST results (DST results available within 31 days of treatment initiation) had a significantly better outcome compared with XDR-TB cases for whom the DST results were available after $>31$ days of treatment [23]. There were 14 XDR-TB patients in the first stratum $(\leqslant 31$ days $)$ and 23 in the second ( $>31$ days) [23]. However, the reasons for this difference were not explained.

Multivariate analysis of factors (demographic, clinical and epidemiological) associated with treatment outcome, which 


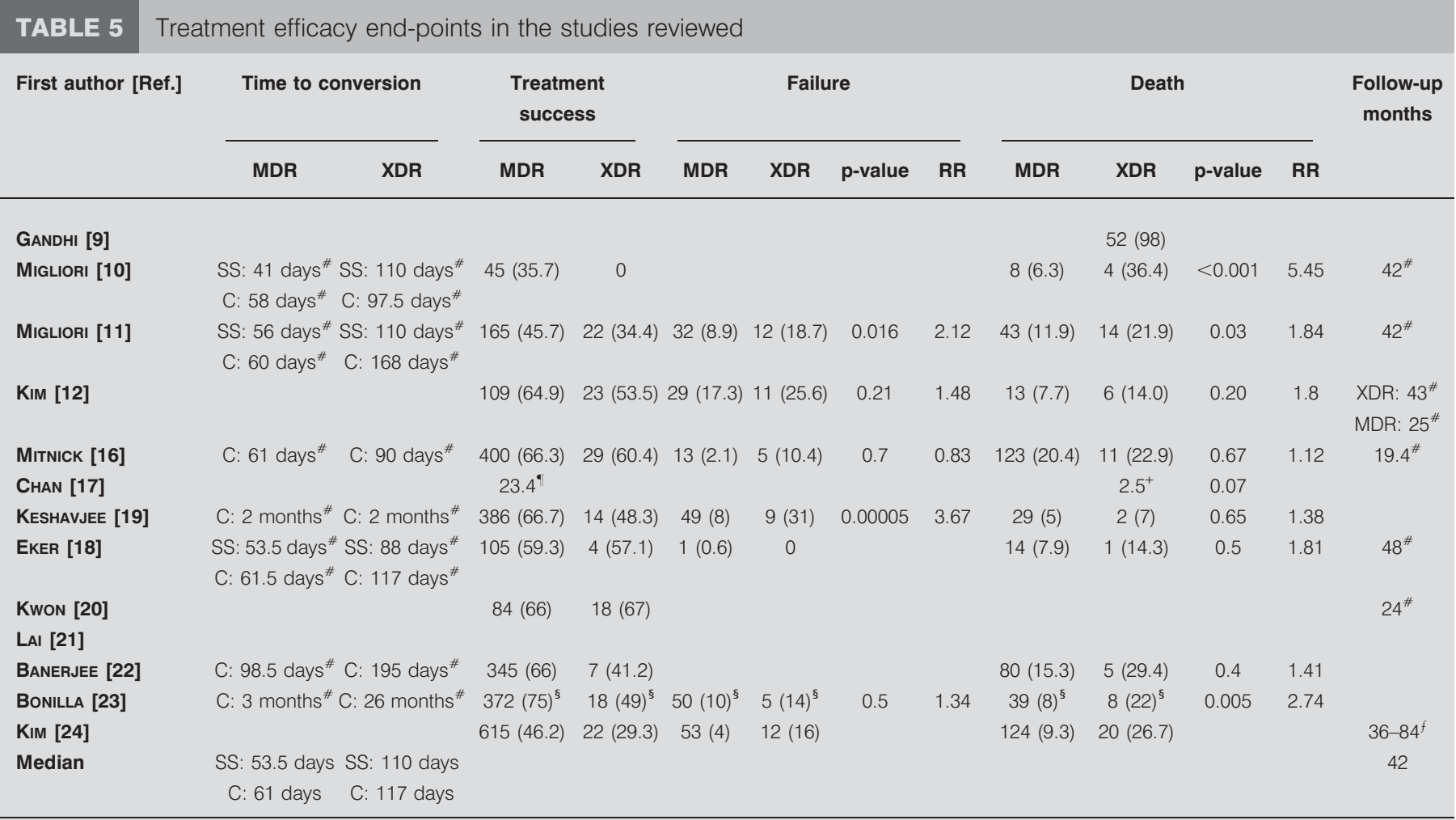

Data are presented as $\mathrm{n}(\%)$, unless otherwise stated. MDR: multidrug-resistant; XDR: extensively drug-resistant; RR: relative risk; SS: sputum smear; C: culture

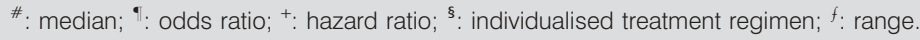

included the XDR-TB status, was performed in three studies $[16,20,24]$. Only one study [24] found a significant association between XDR-TB and poor treatment outcomes (treatment failure or death).

\section{References}

The reference section of only two studies reported all the previously published studies that were included in the present review $[10,11]$.

\section{Summary}

A summary of the variables analysed in the studies reviewed is shown in table 6 .

\section{DISCUSSION}

XDR strains of $M$. tuberculosis have now been identified in at least 49 countries around the globe [30]. After the initial report of an association of XDR-TB with an extremely high mortality in patients co-infected with M. tuberculosis and HIV in a rural area of South Africa in the year 2006 [9], the public suddenly became aware of the threat posed by the emerging drug resistance of $M$. tuberculosis.

The clinical picture of XDR-TB is slowly becoming clearer, with several research groups investigating clinical and epidemiological factors as well as treatment outcomes of patients infected with XDR-TB from different continents. The current authors identified and systematically reviewed 13 studies [9-12, 16-24]. The majority of the studies were published in 2008. The current study is the first systematic review of the clinical epidemiology and treatment outcome of patients with XDR-TB and provides the largest body of consolidated evidence on the topic to date. The results from the present analysis have important implications for understanding the current status of XDR-TB research and for planning future studies that can add to the global body of evidence.

\section{Methodological issues in the study of XDR-TB}

A meta-analysis of the available data was not possible, owing to discrepancies between the studies with respect to definitions used and data elements collected. Since the study of XDR-TB as a phenomenon is relatively new, all the available studies have been affected by one or more of the following pitfalls: retrospective and observational design that does not permit collection of all useful variables; small sample size of XDR-TB cases (range 7-119); lack of quality-assured DST results; variable treatment regimens for XDR-TB (individualised or standardised) that prevent the possibility of drawing any conclusions on the effectiveness of single drugs or drug combinations [9-12, 16, 18-20, 22-24]; and differences in outcome definitions that hamper the ability to compare results across studies (tables 1-3).

Surprisingly, the proportion of retreatment cases in the cohort was not described in some studies and the number of previous treatment regimens longer than 1 month was not described in half of them. The same is true for the number of drugs to which isolates were resistant, which has been shown in some studies to correlate with treatment outcome [10, 11, 16, $18,20]$. All of this information is necessary to describe the 
TABLE 6 Summary of the variables analysed by the 13 studies reviewed

\begin{tabular}{|c|c|c|c|c|c|c|c|c|c|c|c|c|c|c|c|c|c|c|}
\hline \multirow[t]{2}{*}{ First author [Ref.] } & \multicolumn{18}{|c|}{ Variables included } \\
\hline & 1 & 2 & 3 & 4 & 5 & 6 & 7 & 8 & 9 & 10 & 11 & 12 & 13 & 14 & 15 & 16 & 17 & 18 \\
\hline MIGLIORI [10] & Yes & Yes & Yes & Yes & & W & Yes & Yes & Yes & Yes & Yes & & Yes & Yes & Yes & 1 & & \\
\hline MIGLIORI [11] & Yes & Yes & Yes & Yes & & $W / L$ & Yes & Yes & Yes & Yes & Yes & Yes & Yes & Yes & Yes & 1 & & \\
\hline Chan [17] & & & & & & $\mathrm{L}$ & & & & & & & & & & & Yes & \\
\hline KeShavjee [19] & Yes & Yes & Yes & & & L & & Yes & Yes & Yes & Yes & Yes & Yes & & Yes & । & Yes & Yes \\
\hline EKER [18] & Yes & Yes & Yes & Yes & Yes & L & Yes & Yes & Yes & Yes & Yes & Yes & Yes & Yes & Yes & I & Yes & Yes \\
\hline KwON [20] & & Yes & & Yes & & L & Yes & & & & Yes & & & Yes & Yes & 1 & Yes & \\
\hline LAI [21] & & & & & Yes & & & & Yes & & Yes & & & & & & & \\
\hline BANERJEE [22] & Yes & & & & & w & & & Yes & Yes & Yes & & Yes & & & । & & \\
\hline
\end{tabular}

1: representative sample (multidrug-resistant (MDR)-tuberculosis (TB) coverage); 2: history of previous treatment (MDR- versus extensively drug-resistant (XDR)-TB); 3 number with previous treatment for $>1$ month (MDR versus XDR); 4: number of drugs used in treatment (MDR versus XDR); 5: linezolid; 6: standard definitions according to LASERSON et al. [28] (L) and/or the WHO (W); 7: number of drugs to which strains were resistant (MDR versus XDR); 8: 100\% supranational reference laboratory quality assurance drug susceptibility testing; 9: all XDR-defining drugs tested; 10: times to sputum smear and/or culture conversion; 11: treatment success (MDR versus XDR); 12: failure (MDR versus XDR); 13: death (MDR versus XDR); 14: follow-up; 15: prevalence of HIV; 16: standard (S) or individualised (I) treatment; 17: surgery reported; 18 : adverse events reported.

epidemiological context and to correctly interpret the data provided (table 4).

Diagnosis of XDR-TB is one of the most problematic areas of its study. WHO recommendations for second-line DST call for testing one fluoroquinolone and all three injectable agents. However, it is not possible to do so at present in the vast majority of settings with suspected high levels of $M$. tuberculosis drug resistance, because laboratory capacity is lacking and in some cases second-line drugs are not registered for use in the country so DST is not performed. Not all drugs that are included in the current XDR-TB definition were tested in all reviewed studies, as noted. The main consequence is that in several studies the true XDR-TB prevalence may have been underestimated.

DST quality assurance is essential for the diagnosis of MDRand XDR-TB and for surveillance at a national and international level: the WHO and the International Union Against Tuberculosis and Lung Disease are supporting an international network of 26 SRLs that support more than 120 national laboratories [3]. Standardised methods to test second-line drugs were only recently made available, but it is known that current in vitro susceptiblity testing may not be reliable for selected second-line drugs (e.g. cycloserine). Again, this difficulty is hampering the implementation of a fully operational surveillance system for second-line drugs at national level (tables 1-3).

The current authors assessed all the studies selected for the systematic review, recording how many of them reported DST data from quality-assured laboratories [29]. At the time the reviewed studies were completed, second-line drug panel testing had only just been started among the SRLs, and not among national laboratories. So even for laboratories undergoing regular external quality assurance, this may not guarantee the accuracy of the second-line drug susceptibility results reported. Less than half of the studies included in the current review reported DST data obtained from qualityassured laboratories; in other words, only about one patient out of three received a diagnosis of MDR- or XDR-TB (and, most likely, a tailored treatment) based on a DST of proven quality. The current review highlights the many questions still open about the diagnosis and surveillance of drug resistance (table 3).

Most of the studies reviewed reported that patients received individualised treatment based on DST results. However, data were not always available on the number of active drugs used in these regimens. While current expert opinion supports the use of three to five active agents in treating XDR-TB, at least one study reviewed showed successful outcomes with only two to three active agents [20]. Again, more information on methodology would be needed to judge whether these results are reproducible (table 3 ).

The relevant clinical, epidemiological and microbiological differences among the cohorts reviewed are also likely to lower the strength of any conclusions drawn from the treatment efficacy end-point results. Complete evaluation of treatment outcomes as proposed by LASERSON et al. [28] or, alternatively, by the WHO [1] was not described in all studies, highlighting the risk of potential biases in a meta-analysis of the data. Although the WHO definition of cure (based on a negative bacteriological examination in the last month of treatment and on at least one previous occasion) is widely 
used, the definition of LASERSON et al. [28] should be considered the most appropriate to be used in future studies. The latter is, in fact, based on several consistently negative cultures (five) for the final 12 months of treatment and achieved consensus from the main partners in defining cure among MDR-TB patients [28]. As previously stated, the combination of the results of several studies addressing a set of related research hypotheses was not possible (table 2).

When the prevalence of a condition (e.g. MDR- or XDR-TB status) needs to be determined, a sample is considered representative when all cases have the same probability of being included in the sample, and randomisation is the technique allowing minimisation of possible biases. As the main focus of the current review was to discuss the clinical and epidemiological evidence of XDR-TB, the studies enrolling twothirds $(>65 \%)$ of the cases notified in the country were considered representative. This cut-off is valid when discussing clinical aspects (e.g. treatment outcomes, adverse events, etc.), as it excludes extreme selection bias conditions, but it cannot replace the recommended $100 \%$ coverage when the prevalence of the condition needs to be determined (table 1) [2-4, 6].

All of these methodological challenges must be considered when evaluating the results of the studies and formulating new questions for further research.

\section{Treatment issues in XDR-TB}

Treatment of XDR-TB cases relies on drugs that are less potent and much more toxic than those frequently used in the clinical management of disease caused by drug-susceptible or MDR strains. Most reported cases with TB due to XDR strains in the Americas, Europe and Asia have occurred in cases with previous treatment against $\mathrm{TB}$, indicating that former treatment mismanagement may have substantially contributed to the development of drug resistance. Thus, there exists an opportunity for preventing further drug resistance through improved clinical practices.

The role of single drugs or drug combinations in effective XDR-TB treatment has not yet been ascertained. Information on the number and type of effective drugs used was usually not given in the studies reviewed. Most data point to the fact that at least three to four drugs to which the strain is sensitive need to be used for effective treatment, although, in one study from Korea, a high success rate was obtained in spite of a low number of effective drugs (median of two) used in XDR-TB regimens [20]. The XDR-TB regimens used in Peru relied heavily on drugs such as capreomycin, para-aminosalycilic acid and cycloserine, which had not been previously used in the country $[16,23]$. This fact may partially account for the positive results of treatment, in contrast to countries where widespread use of most second-line agents is more common (tables 2 and 3).

In many settings, fluoroquinolones were used in XDR-TB regimens under the assumption that strains resistant to ciprofloxacin may still be sensitive to levofloxacin or moxifloxacin. This assumption has not been proven [31], but the issue of cross-resistance deserves further study to determine the possibility of additional treatment options for patients with early generation fluoroquinolone resistance. Similarly, some studies included isoniazid (at standard or high dose) and rifampicin in XDR-TB treatment regimens because an adequate number of effective drugs were not available [12, 32], but the effect of this strategy was not measured. Finally, most studies included some of the drugs regarded as third-line agents (amoxicillin-clavulanate, clarithromycin, clofazimine, imipenem and linezolid), but their respective contribution could not be ascertained [33].

Surgical resection is a potentially useful strategy for a condition that cannot be adequately controlled with medical interventions. In studies from tertiary hospitals in Korea, $48 \%$ and $56 \%$ of all XDR-TB patients received surgical interventions $[12,20]$ and, in one study, surgery contributed significantly to treatment success compared with cases that did not receive surgery. Even in community MDR-TB programmes like the one in Peru, surgery was used in $14 \%$ of the XDR-TB cases, with a trend towards a better outcome [16]. Surgical resection was performed in $4 \%$ of MDR- and XDR-TB cases in Korea [24]. Surgical treatment improved treatment success in MDR-TB patients $(68.3 \%$ in surgically treated cases versus $44.2 \%$ in nonsurgically treated cases; $\mathrm{p}<0.001$ ). The study was not sufficiently powered to detect a statistically significant difference in patients with XDRTB $(67 \%$ in surgically treated cases versus $28 \%$ in nonsurgically treated cases with $\mathrm{XDR}-\mathrm{TB} ; \mathrm{p}=0.2)$. In the multivariate logistic regression analysis, surgical treatment was the only positive predictor of treatment success among MDR-TB cases $(p=0.001)$, as measured in the overall cohort of MDR-TB cases (not specifically related to XDR-TB cases; table 5).

A crucial question is whether optimal trials to compare efficacy of treatment regimens for XDR-TB can be designed and implemented. Regimens will always need to be individualised and, thus, there will be a large diversity of regimens within a given trial and between trials. Randomisation will not be possible, preventing minimisation of several relevant biases. At a minimum, studies will need to report on the number of effective drugs used on a single patient and stratify by categories of drugs used (first-, second- and third-line). In addition, documenting changes in regimens over time for a single patient can improve interpretation of future study results (table 5).

\section{Outcome issues in XDR-TB}

In contrast to the results presented in the initial report from HIV-seropositive individuals with XDR-TB in South Africa [9], the results of the other studies reviewed suggest emphatically that XDR-TB is not a death sentence for all. With an individually tailored treatment regimen (including pulmonary surgery in selected cases), treatment success can still be achieved in one-half to two-thirds of patients with XDR-TB, as was shown in the majority of studies from the Americas, Europe and Asia.

However, the first reports on XDR-TB confirmed that these patients have, in general, a poorer prognosis and fewer treatment options than MDR-TB cases [10-12]. The majority of the studies analysed in the current review confirmed that XDR-TB has a higher probability of death, failure, longer hospitalisation, longer treatment duration and delayed microbiological (sputum smear and culture) conversion compared with MDR-TB. The fact that mortality was almost invariably increased among XDR-TB cases may suggest that a fraction of 
truly incurable patients does exist, as demonstrated by the first report on this topic (table 4) [10].

Only a few of the available studies (e.g. MitNICK et al. [16] in Peru) reported similar success rates in XDR- and MDR-TB patients. These were obtained despite the fact that XDR-TB patients were on average resistant to a larger number of drugs. A key element, however, is how many effective drugs could be used: this varied significantly within and between studies, although precise figures were not reported and this effect was not analysed (table 4).

Experience from Peru and other countries suggests that strategies to support adherence, as well as psychological, nutritional and even financial interventions, may further contribute to improved outcomes in XDR-TB patients [16, 23, $24]$. The promising results obtained from Korea and Peru also suggest that strict and well-defined rules (e.g. management of XDR-TB cases in specialised reference centres) are essential to improve prognosis. High success rates can also be achieved without hospital care in community MDR-TB programmes [16-24]. Indeed, hospital care may be associated with nosocomial transmission of XDR-TB if adequate infection control strategies are not implemented. The lingering question for further study is whether these encouraging results, achieved with significant effort, can be reproduced in the conditions faced by most TB control programmes worldwide, and what interventions would be needed to achieve high success rates.

With the exception of the South Africa study [9], the studies reviewed reported on cohorts with very low rates of HIV infection and good access to adequate care. Additional data for HIV-infected individuals is needed to determine the role of coinfection in XDR-TB treatment outcome and to evaluate interventions that may contribute to improved outcomes in HIV-infected XDR-TB patients. The high case-fatality rate in the Tugela Ferry outbreak [9] probably represents a combination of factors at play: not only host factors but also lack of access to adequate diagnosis and treatment (table 2).

In short, there are a number of potential factors which may influence treatment outcomes among XDR-TB cases, but the currently available studies were not designed to control for many relevant variables. Further information from other MDRand XDR-TB hot spots (e.g. former Soviet Union countries and the Philippines) is needed to confirm the findings reported in the present review through larger and better-designed prospective studies. An evidence-based approach to this delicate issue should replace the sensationalism that has surrounded XDR-TB in the first 2 yrs following its first description [34].

\section{Conclusion}

According to the criteria used in the current systematic review, 13 studies focusing on clinical epidemiology, management and treatment outcomes of XDR-TB were identified. The results of the systematic review suggest a number of productive areas for further investigation, using improved methods to yield better data.

As with all new topics of investigation, XDR-TB has posed challenges to investigators in optimising their approaches to its study. The current literature cannot form the basis for a metaanalysis because of methodological differences that make comparisons impossible. To strengthen the available body of evidence for the diagnosis and treatment of XDR-TB, and ultimately its prevention, future studies should: 1) be prospective in design; 2) adopt standardised, internationally accepted definitions; 3) use quality-controlled laboratory testing for all first- and second-line drugs that define XDRTB; and 4) collect data on an agreed-upon set of standard variables that will allow for comparison of approaches and results across studies. These variables include, for instance, measures of disease severity (number of drugs to which isolates are resistant and clinical features), treatment history, number and type of active drugs used in treatment, time to sputum smear and culture conversion, treatment duration, adverse events related to treatment, and treatment outcome. Using a systematic approach to the investigation of XDR-TB will yield more useful results more quickly.

A number of important challenges remain, chief among them the speedy and accurate diagnosis of drug resistance. Although rapid, sensitive and specific detection of isoniazid and rifampicin resistance is currently possible, further research is necessary in order to identify new tools able to diagnose resistance to second-line anti-TB drugs in a simple, economical and reproducible manner [35].

Perhaps most importantly, XDR-TB has been shown in the current literature to be a treatable as well as curable condition. While this is an encouraging finding, complacency is not appropriate. XDR-TB patients do more poorly in terms of smear and culture conversion, treatment duration, treatment failure and death. With clear indications that XDR-TB results from mismanaged cases of drug-susceptible and MDR-TB, the first imperative is to treat susceptible $\mathrm{TB}$ appropriately to completion and to provide rapid diagnosis and aggressive, appropriate treatment of MDR-TB to avoid the unnecessary development of additional cases of XDR-TB [5].

More investigation is needed in order to determine optimal treatment regimens using first-, second- and third-line agents (including drugs potentially useful to treat severe XDR-TB cases, such as linezolid), indications for surgery, and other supportive interventions that can increase the chances for treatment success while we wait for new drugs. In addition, more research must be done on XDR-TB and HIV co-infection to avoid a repetition of the devastating results for patients in the Tugela Ferry outbreak [9]. All of this work will require access to large databases from surveillance systems or ad hoc designed studies (e.g. the European-funded FP7 project, TB PAN-NET) with standardised variables on diagnosis, treatment, and outcomes, as double-blind randomised placebocontrolled trials cannot be conducted for ethical reasons.

New diagnostic techniques and new drugs are urgent priorities for adequate control of multidrug-resistant and extensively drug-resistant tuberculosis. At the same time, they can only be effective if introduced into health systems that function adequately to provide access to care and treatment for all of the people who need it, which is another priority that we must continue to address with intensified action. As we move forward on all of these fronts to address the challenges of extensively drug-resistant tuberculosis, early diagnosis and aggressive treatment of extensively drug-resistant tuberculosis can yield positive results for patients and public health. 


\section{ACKNOWLEDGEMENTS}

The authors' affiliations are as follows. G. Sotgiu: Hygiene and Preventive Medicine Institute, University of Sassari, Sassari, Italy. G. Ferrara: Dept of Internal Medicine, Section of Respiratory Diseases, University of Perugia, Perugia, Italy. A. Matteelli: Institute of Infectious and Tropical Diseases, University of Brescia, Brescia, Italy. M.D. Richardson: HIV/ TB Global Program, PATH, Seattle, WA, USA. R. Centis and G.B. Migliori: WHO Collaborating Centre for TB and Lung Diseases, Fondazione S. Maugeri, Care and Research Institute, Tradate, Italy. S. Ruesch-Gerdes: Supranational Reference Laboratory, Research Centre Borstel, Borstel, Germany. O. Toungoussova and A. Spanevello: Fondazione S. Maugeri, Care and Research Institute, Cassano delle Murge, Italy. J-P. Zellweger: TB Clinic, Department of Ambulatory Care and Community Medicine, University of Lausanne, Lausanne, Switzerland. D. Cirillo: Supranational Reference Laboratory, S. Raffaele Institute, Milan, Italy. C. Lange: Division of Clinical Infectious Diseases, Medical Clinic, Research Centre Borstel, Borstel, Germany.

The authors wish to thank L. D'Ambrosio (WHO Collaborating Centre for TB and Lung Diseases, Fondazione S. Maugeri, Care and Research Institute, Tradate, Italy) for her editorial and technical support in development of the current manuscript.

\section{REFERENCES}

1 World Health Organization, Global tuberculosis control surveillance, planning financing. WHO report 2008. WHO/HTM/TB2008.393. Geneva, World Health Organization, 2008.

2 Zignol M, Hosseini MS, Wright A, et al. Global incidence of multidrug-resistant tuberculosis. J Infect Dis 2006; 194: 479-485.

3 Aziz MA, Wright A, Laszlo A, et al. Epidemiology of antituberculosis drug resistance (the Global Project on Anti-tuberculosis Drug Resistance Surveillance): an updated analysis. Lancet 2006; 368: 2142-2154.

4 Espinal MA, Laszlo A, Simonsen L, et al. Global trends in resistance to antituberculosis drugs. World Health Organization/International Union against Tuberculosis and Lung Disease Working Group on Anti-Tuberculosis Drug Resistance Surveillance. N Engl J Med 2001; 344: 1294-1303.

5 Raviglione MC, Uplekar MW. WHO's new Stop TB Strategy. Lancet 2006; 367: 952-955.

6 World Health Organization, Anti-tuberculosis drug resistance in the world. Fourth global report. WHO/HTM/TB/ 2008.394. Geneva, World Health Organization, 2008.

7 Extensively drug-resistant tuberculosis (XDR-TB): recommendations for prevention and control. Wkly Epidemiol Rec 2006; 81: 430-432.

8 Centers for Disease Control and Prevention (CDC)., Emergence of Mycobacterium tuberculosis with extensive resistance to second-line drugs - worldwide, 2000-2004. MMWR Morb Mortal Wkly Rep 2006; 55: 301-305.

9 Gandhi NR, Moll A, Sturm AW, et al. Extensively drugresistant tuberculosis as a cause of death in patients co-infected with tuberculosis and HIV in a rural area of South Africa. Lancet 2006; 368: 1575-1580.
10 Migliori GB, Ortmann J, Girardi E, et al. Extensively drugresistant tuberculosis, Italy and Germany. Emerg Infect Dis 2007; 13: 780-782.

11 Migliori GB, Besozzi G, Girardi E, et al. Clinical and operational value of the extensively drug-resistant tuberculosis definition. Eur Respir J 2007; 30: 623-626.

12 Kim HR, Hwang SS, Kim HJ, et al. Impact of extensive drug resistance on treatment outcomes in non-HIVinfected patients with multidrug-resistant tuberculosis. Clin Infect Dis 2007; 45: 1290-1295.

13 Migliori GB, Lange C, Girardi E, et al. Extensively drugresistant tuberculosis is worse than multidrug-resistant tuberculosis: different methodology and settings, same results. Clin Infect Dis 2008; 46: 958-959.

14 Migliori GB, Lange C, Girardi E, et al. Fluoroquinolones: are they essential to treat multidrug-resistant tuberculosis? Eur Respir J 2008; 31: 904-905.

15 Migliori GB, Lange C, Centis R, et al. Resistance to secondline injectables and treatment outcomes in multidrugresistant and extensively drug-resistant tuberculosis cases. Eur Respir J 2008; 31: 1155-1159.

16 Mitnick CD, Shin SS, Seung KJ, et al. Comprehensive treatment of extensively drug-resistant tuberculosis. $N$ Engl J Med 2008; 359: 563-574.

17 Chan ED, Strand MJ, Iseman MD. Treatment outcomes in extensively resistant tuberculosis. $N$ Engl J Med 2008; 359: 657-659.

18 Eker B, Ortmann J, Migliori GB, et al. Multidrug- and extensively drug-resistant tuberculosis, Germany. Emerg Infect Dis 2008; 14: 1700-1706.

19 Keshavjee S, Gelmanova IY, Farmer PE, et al. Treatment of extensively drug-resistant tuberculosis in Tomsk, Russia: a retrospective cohort study. Lancet 2008; 372: 1403-1409.

20 Kwon YS, Kim YH, Suh GY, et al. Treatment outcomes for HIV-uninfected patients with multidrug-resistant and extensively drug-resistant tuberculosis. Clin Infect Dis 2008; 47: 496-502.

21 Lai CC, Tan CK, Huang YT, et al. Extensively drugresistant Mycobacterium tuberculosis during a trend of decreasing drug resistance from 2000 through 2006 at a Medical Center in Taiwan. Clin Infect Dis 2008; 47: e57-e63.

22 Banerjee R, Allen J, Westenhouse J, et al. Extensively drugresistant tuberculosis in California, 1993-2006. Clin Infect Dis 2008; 47: 450-457.

23 Bonilla CA, Crossa A, Jave $\mathrm{HO}$, et al. Management of extensively drug-resistant tuberculosis in Peru: cure is possible. PLOS ONE 2008; 3: e2957.

24 Kim DH, Kim HJ, Park SK, et al. Treatment outcomes and long-term survival in patients with extensively drugresistant tuberculosis. Am J Respir Crit Care Med 2008; 178: 1075-1082.

25 Migliori GB, Richardson MD, Lange C. Of blind men and elephants: making sense of extensively drug-resistant tuberculosis. Am J Respir Crit Care Med 2008; 178: 1000-1001.

26 Higgins JPT, Green S, eds, Cochrane Handbook for Systematic Reviews of Interventions, Version 5.0.1 (updated September 2008). Oxford, The Cochrane Collaboration, 2008. Available from www.cochranehandbook.org. 
27 Migliori GB, Loddenkemper R, Blasi F, Raviglione MC. 125 years after Robert Koch's discovery of the tubercle bacillus: the new XDR-TB threat. Is "science" enough to tackle the epidemic? Eur Respir J 2007; 29: 423-427.

28 Laserson KF, Thorpe LE, Leimane V, et al. Speaking the same language: treatment outcome definitions for multidrug-resistant tuberculosis. Int J Tuberc Lung Dis 2005; 9: 640-645.

29 World Health Organization, Policy guidance on drugsusceptibility testing (DST) of second-line antituberculosis drugs. WHO/HTM/TB/2008.392. Geneva, World Health Organization, 2008.

30 World Health Organization, Global map on XDR-TB. www.who.int/tb/challenges/xdr/xdr_map_june08.pdf Date last updated: June 2008. Date last accessed: October $28,2008$.

31 Tonkel T, Mishustin S, Strelis A, Yanova G, Chukova N, Yedilbayev A. Treatment outcomes of the use of moxifloxacin in management of patients with XDR-TB in Tomsk Oblast of the Russian Federation. Eur Respir J 2008; 32: Suppl. 52, 227s-228s.

32 Katiyar SK, Bihari S, Prakash S, Mamtani M, Kulkarni H. A randomised controlled trial of high-dose isoniazid adjuvant therapy for multidrug-resistant tuberculosis. Int $J$ Tuberc Lung Dis 2008; 12: 139-145.

33 Condos R, Hadgiangelis N, Leibert E, Jacquette G, Harkin T, Rom WN. Case series report of a linezolidcontaining regimen for extensively drug-resistant tuberculosis. Chest 2008; 134: 187-192.

34 Migliori GB, Cirillo DM, Spanevello A, Codecasa LR, Stop TB Italia Group, Ripped from the headlines: how can we harness communications to control TB? Eur Respir J 2007; 30: 194-198.

35 Ling DI, Zwerling AA, Pai M. GenoType MTBDR assays for the diagnosis of multidrug-resistant tuberculosis: a meta-analysis. Eur Respir J 2008; 32: 1165-1174. 